University of Toronto Neurosurgical Rounds No. 2.

\title{
Oculodermal Melanosis (Naevus of Ota) Complicated by Multiple Intracranial Tumors
}

\author{
W.J. HORSEY, J.M. BILBAO, J. NETHERCOTT, R. MYERS and H.J. HOFFMAN
}

This case was presented as an example of a rather rare disease. Apart from its unusual features, it leads one to reflect on pigmentation and its relationship to neoplasia.

\section{CASE HISTORY}

A thirty-seven year old, East Indian female was admitted to St. Michael's Hospital on July 26, 1979 complaining of headache, diplopia, nausea and vomiting.

In April of 1978 she was referred to a neurologist for investigation of headache. Neurological examination was normal. An X-ray of her skull revealed a lytic lesion, $2 \mathrm{cms}$. in diameter, in the right frontal area. A bone scan showed no abnormality. Further study was refused. A left facial "birth mark" was noted.

While visiting in India in July 1979 , she complained of generalised headache of increasing severity. She had double vision with nausea and vomiting on occasion. She flew back to Canada and was admitted to hospital.

Examination revealed a well nourished, drowsy, East Indian female who was slightly confused and disorientated. Even after her level of consciousness had improved following the administration of dexamethazone, she remained withdrawn and reluctant to discuss her symptoms. She was fearful and occasionally tearful. She had bilateral papilledema with hemorrhages. Bilateral sixth nerve palsies were noted. There were no other neurological signs. The heart rate was 60 per minute and the blood pressure $120 / 65 \mathrm{~mm}$. of mercury.

She had a bluish-brown discolora-

From the Departments of Neurosurgery, Neuropathology and Dermatology, University of Toronto.

Reprint requests to: W. J. Horsey. MD. St. Michael's Hospital, 30 Bond Street, Toronto, Ontario, M5B IW8, Canada. the ophthalmic and mandibular divisions. The conjunctiva on the left was of a deep, blue-black hue (Fig. 2) while there was paler staining of the mucus membrane of the cheek and hard palate. The lesion was identified as a naevus of Ota (Ota, 1939).

Bilateral carotid angiography revealed a shift of the left anterior cerebral artery to the right in its anterior portion and splaying and elevation of the terminal branches of the middle cerebral artery. In the venous phase, a rounded mass was identified in the left parietal area.

A CT scan with enhancement confirmed the presence of a mass close to the surface in the left parietal region. There was evidence of central cavitation of the lesion and edema of the adjacent brain. (Fig. 3). In a lower cut, there was a second mass in the region of the apex of the petrous portion of the left temporal bone. It lay partly in the posterior fossa and partly in the middle fossa. (Fig. 4). Although there was displacement of the lateral ventricles and the third ventricle to the right, there was no ventricular dilatation. (Fig. 5). We concluded that the large, supratentorial mass in the left parietal area was responsible for the elevated intracranial pressure. The CT scan failed to disclose any abnormality in the region of the radiolucent zone in the right frontal region.

With a presumptive diagnosis of intracranial metastatic malignancy, the left hemisphere mass was exposed through a parietal craniotomy. The shaved scalp appeared normal. When the skin was incised the cut surface was unremarkable. The exposed periosteum, however, was darkly pigmented. When the osteoplastic flap was raised, there was obvious pigmentation of the diploe between layers of normal looking compact bone. (Fig. 




Figure I - Left profile of patient. Note pigmented areas which are blue-black in colour.

6). The outer surface of the exposed dura mater was black and on opening it, its full thickness was pigmented (Fig. 7). There were small scattered areas of brown discoloration of the cortex. A somewhat lobulated, black tumor mass, loosely adherent to the dura was found indenting the parietal lobe. The adjacent brain was stained light brown. It was possible to develop a plane between the lesion and the brain and to remove it with minimal damage to the cerebral substance. The portion of dura attached to the tumor was excised. After the mass had been shelled out, it was possible to displace the brain to inspect the dura for several centimeters beyond the limits of the craniotomy. As far as one could see the dura was black. An attempt was made to expose the tumor at the left petrous apex but it was not possible through the existing opening.

Numerous biopsies of the pigmented tissues were obtained during the procedure. The dural defect was closed

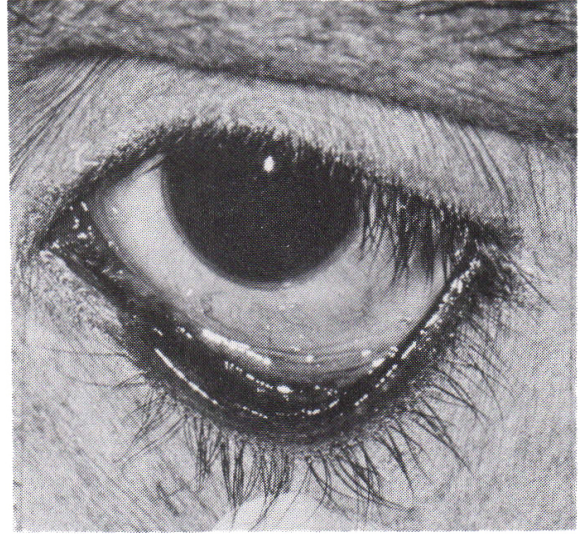

Figure 2 - Left eye of patient with lid everted to display pigment of conjunctiva.

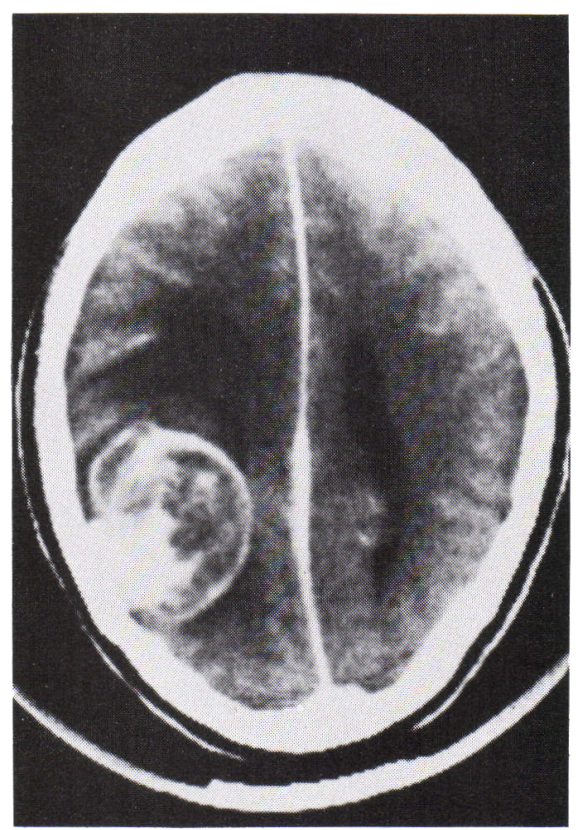

Figure 3 - CT scan through left parietal tumor.

with a piece of periosteum (albeit pigmented) and the wound was closed in the usual manner.

At the time of operation, we thought we were dealing with a malignant melanoma of unknown source. There was no evidence of melanoma in the eyes or in the orbits. A search for cutaneous malignant melanoma was unrewarding.

Post-operatively, the patient complained less of headache. She was more communicative. Her bilateral lateral rectus palsies improved. She was discharged from hospital on the tenth 


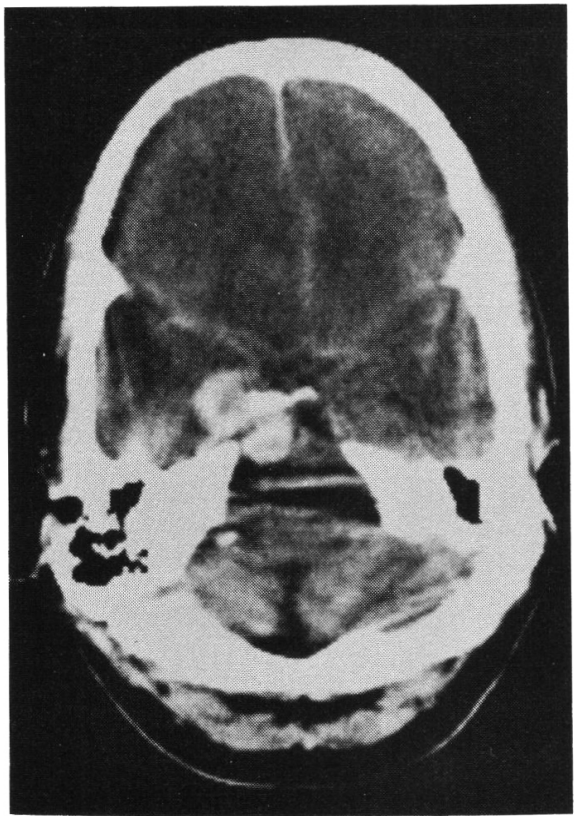

Figure $4-C T$ scan through tumor at the apex of the left petrous pyramid.

post-operative day. When seen about a month later, there was no diplopia and the papilledema had disappeared. She received a course of radiation therapy to her skull.

Six months after operation she was symptom-free. A CT scan at that time revealed no evidence of the left parietal tumor and the lesion at the left petrous apex was unchanged.

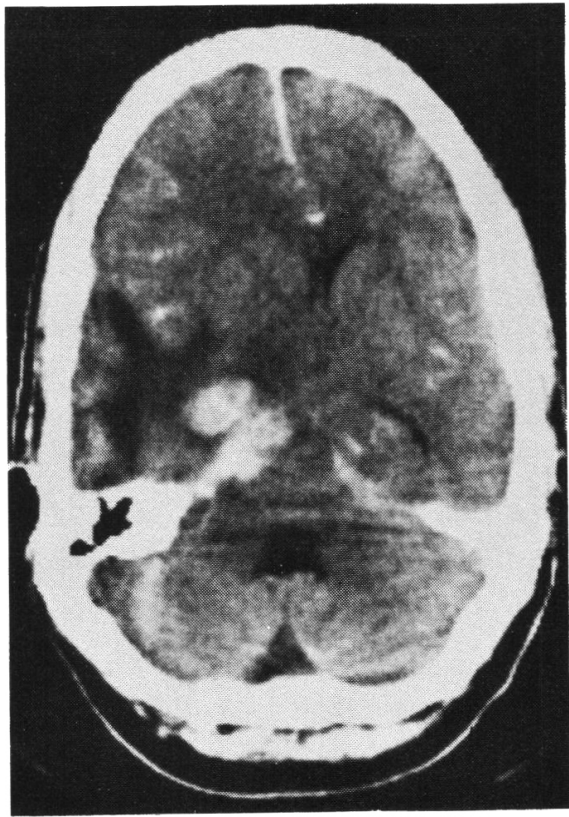

Figure 5 - CT scan showing displacement of the ventricular system to the right. There is no ventricular dilatation.

\section{DISCUSSION}

\section{Dr. W.J. Horsey}

This patient exhibits a number of rather unusual features. The first is the naevus of Ota or oculodermal melanosis, a form of blue naevus. Typically, this is encountered in the yellow races, but it occurs in others in whom there may be a higher incidence of associated malignancy occurring characteristically in the orbit, the eye, and the skin (Speakman and Phillips, 1973). Although Ota's classic description did not appear until 1939, the lesion had been previously recognised and in 1861, Hulke described an orbital malignancy in a patient with a facial naevus typical of oculodermal melanosis. Since that time, sporadic cases have been reported principally in the dermatological and ophthalmological literature. A similar condition involving the shoulder area was described by Ito in 1954 .

Reports of only five cases of naevus of Ota complicated by malignant melanotic intracranial tumors could be found. The first case of associated cerebral melanocarcinoma was reported by Willis (1948) in his textbook entitled "The Pathology of Tumors". The description of the naevus on the face is not detailed, but it is reasonable to infer that the patient had a naevus of Ota. In 1959, Kojima described a patient with oculodermal melanosis and a melanotic tumor involving the optic chiasm. In 1965, Gomez-Gonzalez et al reported a typical case of intracranial malignant melanoma in a patient with Ota's naevus. Enriquez et al (1973) reported a patient with pineal and meningeal implants of melanoma. At autopsy there were no cutaneous or other

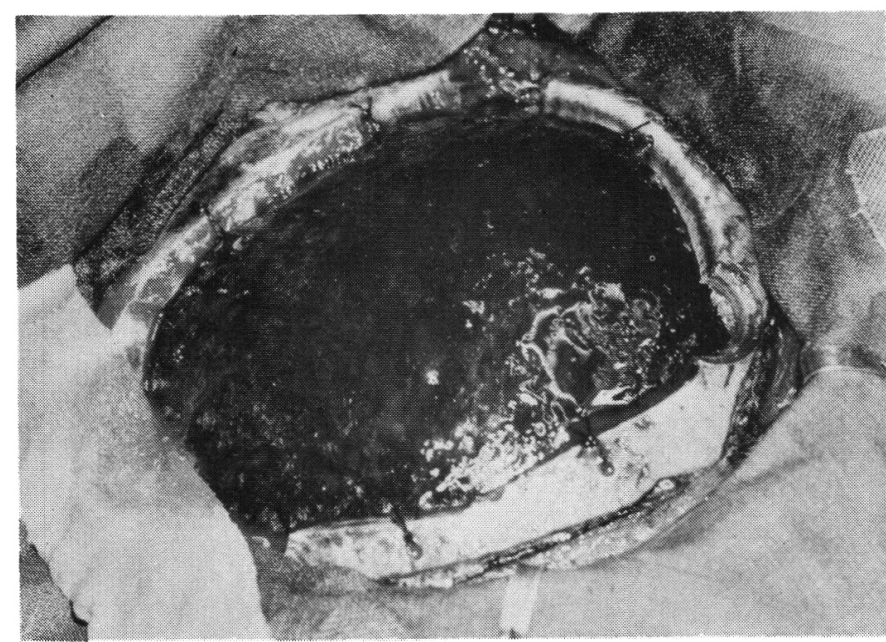

Figure 6-Operative exposure prior to opening of the dura mater. Note: deep pigmentation of the dura, of the diploe, and the periosteum.



Figure 7 - Operative exposure after opening of the dura mater showing the dark tumor and some spotty pigmentation of the cortex. The patty defines the plane between the tumor and the brain. 


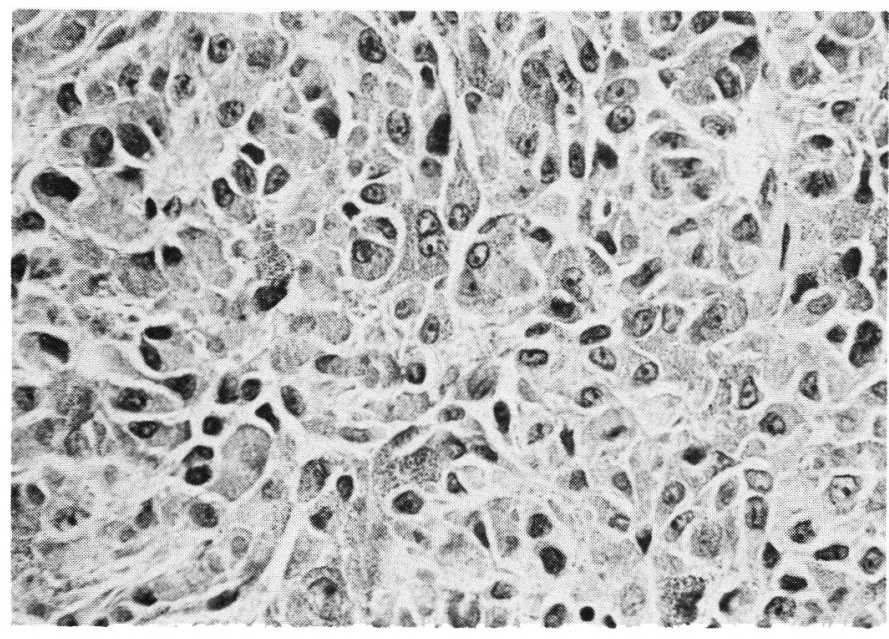

Figure 8-Bleached paraffin section of the tumor showing sheets of slightly irregular polygonal cells with defined outlines (H \& E mag. $\times 250$ ).

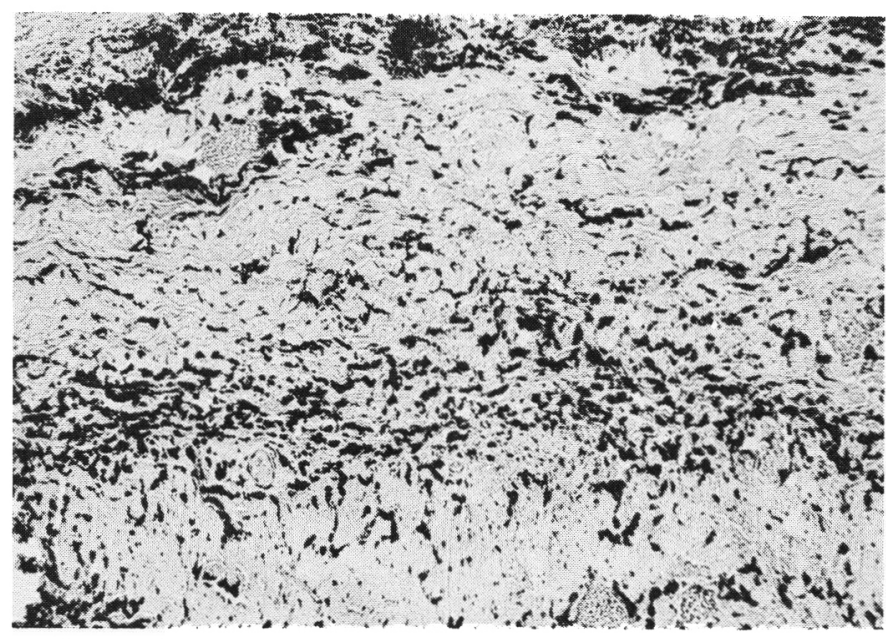

Figure 9 - Section of dura mater showing melanin-laden cells with slender cytoplasmic extensions reminiscent of dendritic processes of melanocytes. (H \& E mag. x 100). deposits of melanocarcinoma. In 1977 , Sang et al described a case of naevus of Ota with a malignant melanoma on the opposite side of the brain.

\section{Dr. J. Bilbao}

There are two aspects of this case that will be discussed. One is the tumor itself and the other is the pigmentation of the adjacent tissues, namely the dura mater, bone and periosteum.

When the subdural tumor mass was sectioned it oozed blackish fluid indicating necrosis within the tumor. It was difficult to find areas for histological examination. The tumor was attached to a small piece of dura mater. Paraffin sections showed most of the tumor cells so heavily pigmented that cytological detail was not discernible. Upon bleaching the paraffin sections the tumor appeared highly cellular and composed of sheets of slightly irregular polygonal cells with defined outlines. (Fig. 8). They exhibited discrete nuclear pleomorphism and rare mitotic figures. The diagnosis was that of melanocarcinoma in spite of the absence of overt cellular atypia. Several pieces of brain from the tumor bed showed gliosis as well as some pigment in the perivascular spaces incorporated in the histiocytes, but there was no histological evidence of infiltration of brain by tumor.

Sections of the dura mater and periosteum showed a large number of melanin-laden cells with small nuclei, slightly granular cytoplasm and slender cytoplasmic extensions reminiscent of dendritic process of melanocytes. (Fig. 9). This lesion was interpreted as being a blue naevus of the dura mater and periosteum. Focal infiltration by tumor cells of the dura mater was confined to the site of attachment of the tumor.

The bone seen under the dissecting microscope showed a brownish-black reticulate representing pigment in the Haversian channels. Bleached paraffin sections of the skull showed the presence of non-neoplastic pigmentladen cells in the Haversian channels with preservation of the structures of bone.

Normally, aggregates of melanocytes are found in the pia mater particularly along the ventral surface of the medulla and upper cervical cord, and perhaps it is for this reason that primary meningeal melanocytomas are mostly found in the areas of the posterior fossa, foramen magnum and the spinal cord (Steinberg et al, 1978).

Neurocutaneous melanosis is a congenital dysplasia of the neural crest in which diffuse and often invasive proliferation of melanin-containing cells occur throughout the leptomeninges. It is seen mostly in infants under two years of age, who also exhibit extensive nevi of the trunk or shoulder area. (Slaughter et al, 1969). These cases should be distinguished from the naevus of Ota in that the pigmentation here is confined to the dura mater, periosteum and bone, pigmentation of the leptomeninges being minimal. This perhaps reflects the fact that the pigmentation in the naevus of Ota develops in the distribution of the trigeminal nerve. A number of cases have appeared in the literature describing the association of naevus of Ota with melanoma of the choroid (Albert and Scheie, 1963), orbit (Hagler and Brown, 1966), ciliary body (Hadasa, 1970) and less frequently brain (Sang et al, 1977) and pineal gland (Enriquez, 1973).

\section{Dr. J. Nethercott}

Named cutaneous disorders associated with proliferation of melanocytes are numerous. The most common forms are well known. Table 1 lists these.

The patient presented here has a disorder of melanocyte proliferation in the dermis. These disorders are referred to as dermal melanoses. In these, there is an arrest of melanocytes derived from Schwann cells in the dermis. This group of disorders includes the Mongolian spot, blue naevi (common and cellular type), naevus of Ota, and naevus of Ito.

Mongolian spots are areas of pigmentation, usually over the sacrum, which are found in $90 \%$ of Mongolian children at birth. Such pigmented areas are also found in people of Finnish and Hungarian origin as well 
TABLE ।

Disorders of Melanocytic Proliferation

\section{Lentigo}

Melanocytic Naevi

Common:

\section{Junctional}

Compound

Intradermal

Special Types:

Oculocutaneous

Congenital

Giant Hairy

Neurocutaneous Melanosis

Juvenile Melanoma

Dermal Melanocytosis

Mongolian Spot

Blue Naevus

Ota's Naevus

Ito's Naevus

Malignant Melanoma

Epidural Origin:

Lentigo Maligna

Superficial Spreading

Nodular

Dermal Origin:

Malignant Blue Naevus

as in polynesian and negro infants. The lesion is present at birth and usually resolves by adolescence (Fitzpatrick and Mihm, 1971).

Blue naevi are pigmented tumors usually presenting on the distal portion of the extremities. Based on the experience of Dorsey and Montgomery (1954) of 500 blue naevi seen over a two-year period in the Mayo Clinic, it is reasonble to assume that this is a fairly common dermal melanocytic disorder. It may be more common in females. Usually, these lesions are biopsied or excised because they are confused with primary malignant melanomas. Two types are recognised. The first is known as a common blue naevus, which has small dendritic cells scattered through the connective tissue. The other, known as cellular blue naevus, is characterised by plumper cells which begin to aggregate in clusters in the midst of the other cells. Common blue naevi outnumber cellular blue naevi by ten to one. Some- times, the plump cells of cellular blue naevi are found in draining lymph nodes. This has not been interpreted as metastasis but rather that such cells migrate to the lymphatics because they are squeezed out of the rapidly dividing area of naevoid growth (Allen and Spitz, 1953).

Common blue naevi are not considered to have significant potential to undergo malignant transformation. In contrast, cellular blue naevi occasionally undergo change into malignant blue naevi. In instances where common blue naevi have undergone malignant transformation, usually an area of transformation into cellular blue naevus can be found in the common blue naevus (Rodriguez and Ackerman, 1968).

Proliferation of dermal malanocytes occurring in a dermatomal distribution has been recognised since 1939 , when Ota, a Japanese physician described some Japanese children who had developed slate-grey pigmentation in the distribution of the first and second divisions of the trigeminal nerve in early childhood. He described scleral pigmentation as part of the syndrome. Often this pigment proliferation does not affect the sclera. Sometimes the mucus membrane of the mouth is involved (Hidano et al, 1967). By the early 1950's, the Japanese had described over 500 cases of this disorder which has become known as naevus of Ota (Ito and Yoshida, 1952). More than 60 cases have been reported in Caucasians.

Development of a slate-gray patch of hyperpigmentation associated with dermal melanocytic proliferation in the area of the shoulder or upper back was described in a group of children by the Japanese physician Ito (Ito and Yoshilda, 1952) and this is now referred to as a naevus of Ito.

The patient presented here exhibits slate-gray pigmentation principally in the second division of the trigeminal nerve but also involving the first and third divisions. The affected areas have been tattooed with a flesh-coloured pigment, which made it less noticeable. There is no scleral involvement. Histopathological analysis reveals the presence of dendritic cells, as found in the common blue naevus, not only involving the skin of the patient but also involving the bone and the nervous system. In contrast to patients previously reported with naevus of Ota who developed melanocarcinoma with a focus of cellular blue naevus cells, our patient appears to have had invasion of the nervous system in keeping with melanocarcinoma without any focus of cellular blue naevus cells having developed.

Silverberg et al (1971) reported a patient with cellular blue naevus of the scalp showing invasion of the calvarium and brain. The patient experienced a long period of survival after surgical extirpation of the lesion involving the central nervous system. Thus, the prognosis for survival in the case presented here may not be dismal.

\section{Dr. R. Myers}

In discussing this lady's general management I will be practical. My plan was to deal with this as a cerebral secondary tumor because there were two lesions on the CT scan. I think there is some debate as to whether this is a secondary and there may be debate as to whether it is malignant. Doctor Bilbao feels it is malignant and the fact that there are two lesions supports the opinion that they may be metastases, recognising that no primary lesion is visible. It is known that about $5 \%$ of cases with melanoma will have an occult primary lesion. The approach to multiple metastatic intracranial melanoma is similar to that of other intracerebral metastases. All patients receive steroids and if indicated intravenous mannitol is used and occasionally a shunting procedure is needed. When the tumors are multiple radiation is the only treatment known to have any effect. If the disease is under excellent control, surgical excision of a solitary metastasis may be helpful. In this case, despite the fact that there were two lesions, surgery was needed to establish the diagnosis and to relieve symptoms caused by the tumor which was removed.

The results of surgical treatment of cerebral metastases have been well reviewed in a number of different series throughout the years. It must be appreciated that these were selected patients who were in relatively good health and who usually presented with 
a solitary lesion. The post-operative mortality at about one month varied from as high as $32 \%$ to as low as $4 \%$. However, the outlook in this situation was bleak with a median survival varying from about four to six months, while the survival at one year was about 25\%. (Posner, 1977).

The results with radiation therapy of cerebral metastases have also been reviewed. Early mortality is only mentioned in a couple of series and is generally about $10 \%$. The median survival was poor, being about six months, although Posner (1977) reported a median survival as low as fifteen weeks. Gottleib et al (1972) reported specifically on a series of 41 patients with metastatic melanoma to the brain who were treated with radiation and in whom median survival was only three months.

Although tumors from lung and breast give rise to a higher absolute number of cerebral secondaries, autopsy series suggest that patients with melanoma have a $50-75 \%$ likelihood of developing cerebral secondaries and in these situations $75 \%$ of them are multiple. (Patel et al, 1978).

From a study at the Princess Margaret Hospital, Toronto, of patients with melanoma and cerebral metastases, the median survival was 11.7 weeks, while only $25 \%$ of the patients survived at 32.8 weeks. (Keen, 1979). Hence, the outlook for patients with cerebral secondaries in general and metastases from melanoma is poor.

At the Princess Margaret Hospital there has been an additional study of patients with cerebral metastases of all sorts, comparing conventional radiation to a single dose of $1,000 \mathrm{rads}$ (Harwood and Simpson, 1977). It was found that both types of treatment were followed by almost the same survival time and, perhaps more significantly, the improvement of symptoms was about the same. In addition, further experience with melanoma, long considered to be radioresistant, suggests that it does respond to radiation therapy. In tissue culture treated by a single large dose of radiation, it has been shown that there has been a larger cell kill than that seen when conventional radiation is used. (Harmer, 1976).
In the patient under discussion, as there was one cerebral lesion left behind following operation, we felt that cranial radiation was indicated. For some of the reasons mentioned, we elected to treat her with a single dose of 1,000 rads to her entire brain. This was tolerated well. In addition, we carried out careful studies to rule out the presence of disease in other organs. As these results were negative, we did not think that systemic chemotherapy was indicated.

If systemic disease is identified, treatment with the chemotherapeutic agent 5-(3,3-Dimethyl-I-Triazeno) Imidazole-4-Carboximide will probably be instituted. This is known to be effective in $20-30 \%$ of patients with metastatic malignant melanoma.

\section{Dr. H. Hoffman}

We recently had an interesting patient with a similar lesion at the Hospital for Sick Children. This 7 year old girl was referred to Doctor Richard Cohen, a plastic surgeon in St. Catharines, Ontario because of a large blue naevus in the right frontoparietal region of her scalp. The naevus was flat with an indistinct border and measured about $8 \mathrm{~cm}$. in diameter. However, in the central portion of the naevus there was a very dark blue area which was raised and measured $1.5 \mathrm{~cm}$. in diameter. Over a 4-week period, this central portion doubled in size. Because of this rapid increase, Doctor Cohen attempted excision of the lesion. However, at operation he found that the naevus penetrated full thickness of scalp and went down to the underlying temporalis muscle. The tissue biopsied by Doctor Cohen was submitted to the National Cancer Institute who sent it to the various authorities. Doctor Mihm at the Massachusetts General Hospital felt that it was a highly dysplastic congenital naevus which was probably malignant. Doctor H.W. Clark of the University of Pennsylvania felt that the lesion was a spindle-cell malignant melanoma. Doctor McCaughey of the National Cancer Institute felt that the lesion was a benign blue naevus.

Doctor Cohen referred the patient to us because of the possibility that it might be malignant and seemed to be invasive.

A CT scan showed bony expansion and lysis in the anterior portion of the right parietal bone which was interpreted as indicative of bony destruction by an infiltrating melanoma.

Considerable discussion subsequently ensued as to what to do about it. Doctor H. Thomson of our plastic surgical service thought that there had never been a malignant blue naevus at the Hospital for Sick Children.

On August 20, 1979, at the age of $71 / 2$ years, she was admitted. On August 21, her lesion was excised. The melanoma went through all layers of scalp to underlying temporalis muscle, through blackened skull and through the outer layer of dura. The inner layer of dura was devoid of melanoma cells. All of the involved tissue including scalp, muscle, periosteum, skull and dura were excised. The dural defect was patched with human freeze-dried dura. The bony defect which was the size of two silver dollars was repaired with a tantalum mesh acrylic cranioplasty. Doctor H. Thomson then rotated in a scalp flap and used split thickness skin to repair the scalp defect.

Post-operatively, she did well and was discharged from hospital ten days later.

Pathological examination of the tissue revealed a benign melanoma which was present in scalp, muscle, periosteum, skull and dura. We felt that the scalp blue naevus had invaded the deeper tissues. Silverberg et al (1971) reported a similar case in which a benign blue naevus had invaded through skull and dura into brain directly. Menter et al (1971) reported a case similar to ours.

It appears that these large cellular blue naevi of scalp can invade deeply and if not resected, can eventually involve underlying brain leading to significant neurologic deficit. In view of the benign nature of these tumors, early total excision seems desirable and is possible.

\section{Dr. Horsey}

This differs from our case and the cases in the literature with malignant brain tumors. It leads one to reflect on 
the concepts of melanosis, neoplasia and malignancy. Were you able to get beyond the area of dural pigmentation on all sides?

\section{Dr. Hoffman}

Yes.

\section{Dr. Horsey}

In our patient, I tried to reach the second tumor at the base of the skull, but I was unable to expose it. Nevertheless, I had an excellent view of the dura mater over much of the convexity of the left cerebral hemisphere and of the tentorium cerebelli. There was no area of exposed dura which was not deeply pigmented. This would appear to be a unique feature in our case.

\section{Question}

Can this condition be confused with neurocutaneous melanosis?

\section{Dr. Horsey}

Giant naevus or neurocutaneous melanosis must be distinguished from the naevus of Ota. As pointed out by Doctor Bilbao, the former condition is found in children. There is a giant hairy naevus, often with a cape or bathing-trunk distribution. Commonly, it is associated with hydrocephalus, raised intracranial pressure, chronic basal meningitis, cranial nerve palsies, and retardation.

\section{Dr. T. Morley}

What is the relationship between these tumors and pigmented meningioma?

\section{Dr. Bilbao}

Perhaps the term "pigmented or melanotic meningioma" should be dropped altogether. With the electron microscope it can be shown that these tumors are actually cellular blue naevi.

\section{Dr. Harley Smyth}

Was there a family incidence in the cases reported in the literature?

\section{Dr. Horsey}

Most of the patients with malignant change are described as single case reports without reference to the families. Although familial occurrence of the naevus of Ota is described, it is not considered an inheritable one. (Dodd, 1977).

\section{Question}

Is it possible to diagnose intracranial malignant melanoma by examination of the spinal fluid?

\section{Dr. Horsey}

Yes, it certainly can be done, but it would have been very hazardous to carry out a lumbar puncture on this patient with a large supratentorial mass and raised intracranial pressure. Malignant melanin-containing cells have been found in the spinal fluid of other patients, and brown discoloration of the spinal fluid has been reported.

\section{REFERENCES}

ALBERT, E.M., SCHEIE, H.C. (1963). Naevus of Ota with malignant melanoma of the choroid. Arch. Ophthalmol. 69: 774-777.

ALLEN, A. and SPITZ, S. (1953). Malignant Melanoma. Cancer, 6: 1-45.

DODD, W.A. (1977). Naevus of Ota and naevus of Ito. Clinical Dermatology, Volume 2. Dennis, D.J., Dodson, R.L., and McGuire, J. (Ed.) Unit 11-45: 1-4. Harper \& Row Publishers, Inc. Hagerstown, Maryland.

DORSEY, C.. and MONTGOMERY. $\mathrm{H}$. (1954). Blue naevus and its distinction from Mongolian Spot and the naevus of Ota. J. Invest. Derm. 22: 225-236.

ENRIQUEZ, R., EGBERT, B., BULLOCK, J. (1973). Primary malignant melanoma of central nervous system. Arch. Path. 95: 392-395.

FITZPATRICK, T., and MIHM, M. (197I). Abnormalities of the melanin pigmentary system. Dermatology in General Medicine. McGraw-Hill, New York.

FONT, R.L., REYNOLDS, A.M., ZIMMERMAN, L.E. (1967). Diffuse malignant melanoma of the iris in the naevus of Ota. Arch. Ophthalmol. 77: 513-518.

GOMEZ-GONZALEZ, J., ROSELLI, A., RODRIGUEZ, A. (1965). Melanosis oculodermica, melanoblastosis leptomeningea and melanoma intracerebral primario. Univ. Med. 7: 47-52.

GOTTLIEB, J.A., FREI, E., LUCE, J.K. (1972). Evaluation of the management of patients with cerebral metastases from malignant melanoma. Cancer, 29: 701-705.

HADASA, A. (1970). Malignant melanoma in a case of bilateral naevus of Ota. Arch. Ophthalmol. 84: 176-178.

HAGLER, W.S., BROWN, C.C. (1966). Malignant melanoma of the orbit arising in naevus of Ota. Trans. Am. Ac. Ophthalmol. \& Otol., 70: A-17, A-22.

HARMER, C.L. (1976). The radiotherapy of melanoma. Clinical and Experimental Dermatology. I, 29.
HARWOOD, A.R. and SIMPSON, W.J. (1977). Radiation therapy of cerebral metastases: A randomized prospective clinical trial. Int. J. Radiation Oncol. Biol. Phys. 2: 109!-1094.

HIDANO, A., KAJIMA, H., MIZUTANI, MIYASATA, $H$. , and NIIMURA, $M$. (1967). Natural history of naevus of Ota. Arch. Derm. 95: 187-195.

HULKE, J.W. (1861). A series of cases of carcinoma of the eyeball. Ophthalmol. Hosp. Rep. 3: 279-286.

ITO, M. (1954). Naevus fusco-caeruleus. Tohoku. J. Exp. Med. 60: 10.

ITO, M. and YOSHIDA, K. (1952). Studies on melain; VIl naevus fusco-caeruleus ophthalmo-maxillaris. Ota, Tohuko. J. Exper. Med. (Suppl. 1) 55: 34-43.

KEEN, C. (1979). Personal communication.

KOJIMA, K., NATSUME, C., HOUDA, A. et al (1959). A melanoma of the optic chiasm occurs in a case of Ota's naevus and melanosis bulbi. Jap. J. Clin. Ophthalmol. 13: $502-504$.

MENTER, M.A., GRIESSEL, P.J.C., and deKLERK, D.J. (1971). Giant blue naevus of the scalp with underlying skull defect. Br. J. Derm., 85: 73-75.

OTA, M. (1939). Naevus fusco-caeruleus ophthalmo-maxillaris. Jap. J. Dermatol. 46: 369.

PATEL, J.K., DIDOLKAR, M.S., PICKREN, J.W., MOORE. R.H. (1978). Metastatic pattern of malignant melanoma. Am. J. Surg. 235: 807-810.

POSNER, J.B. (1977). Management of central nervous system metastases. Seminars in Oncology. 4: 81-91.

RODRIGUEZ, H., and ACKERMAN, L. (1968). Cellular blue naevus - clinicopathological study of forty-five cases. Cancer, 21: 393-405.

SANG, D.N., ALBERT, D.M., SOBER, A.J., MCMEEKIN, T.O. (1977). Naevus of Ota with contralateral cerebral melanoma. Arch. Ophthalmol. 95: 1820-1824.

SILVERBERG, G., KADIN, M., DORFMAN, R., HANBERY, J. and PROLO, D. (1971). Invasion of the brain by a cellular blue naevus of the scalp. A case report with light and electron microscopic studies. Cancer, 27: 349-355.

SLAUGHTER, J.C., HARTMAN, J.M. and KEMPE, L. et al (1969). Neurocutaneous melanosis and leptomeningeal melanomatosis in children. Arch. Pathol. 88: 298-304.

SPEAKMAN, J.S., PHILLIPS, M.J. (1973). Cellular and malignant blue naevus complicating oculodermal melanosis. Can. J. Ophthalmol. 8: 539-547.

STEINBERG, J.M., GILLESPIE, J.J.. MACKAY, B., BENJAMIN, R. and LEAVENS, R. (1978). Meningeal melanocytoma with invasion of the thoracic spinal cord. J. Neurosurg. 48: 818-824.

WILLIS, R.A. (1948). Pathology of Tumors. London, Butterworth \& Co. (Publishers) Ltd. pp. 913-914. 